\title{
Nebenwirkungen von Medikamenten an Muskel, Gelenk und Nerv - Drei Fallberichte
}

Schwäche in den Oberschenkeln, Hüftschmerzen, Sensibilitätsstörungen. Drei Fallbeispiele zeigen die Komplexität der Nebenwirkungsproblematik neuromuskuloskeletaler Beschwerden. Erst der Weg über mehrere Arbeitshypothesen bringt die wahre Ursache der Beschwerden zum Vorschein - ein Medikament. Vor allem wenn der Behandlungsverlauf nicht einem erwarteten Muster entspricht, sollten Manualtherapeuten sich die Arzneimittel der Patienten genauer ansehen.

Manualtherapeuten sind spezialisiert auf neuromuskuloskeletale Befunderhebungen und Behandlungen. Die Kenntnis der Medikation des Patienten ist dabei unabdingbar für das Clinical Reasoning unter Einbezug der ICF-Kontextfaktoren. Der ärztliche Unterricht, der in der Weiterbildung Manuelle Therapie vorgeschrieben ist, zielt auch auf den Erwerb von Kenntnissen über Medikamente und ihrer Relevanz im manualtherapeutischen Setting [9]. Der Therapeut wird bei der Erhebung der Medikamentenanamnese mit Arzneimitteln konfrontiert, die für das neuromuskuloskeletale Problem des Patienten vom Arzt verordnet, vom Patienten selbst gekauft oder aus dem von allen Familienmitgliedern gespeisten häuslichen Bestand genommen wurden. Er sollte die Medikamente des Patienten auch als Ursache für dessen aktuelles neuromuskuloskeletales Problem in Erwägung ziehen, insbesondere dann, wenn das Problem nicht einem erwarteten Muster entspricht oder einen protrahierten Verlauf zeigt. Informationen zu Nebenwirkungen stehen in der Gebrauchsinformation (= Beipackzettel) oder in der im Internet zu findenden wissenschaftlichen Fachinformation. Nebenwirkungen und deren Häufigkeit sind seit 1995 auch in der Nebenwirkungsdatenbank des Bundesinstitutes für Arzneimittel und Medizinprodukte (BfArM) [4] und seit 2007 in der Datenbank „sanego“ [13] zu finden. Die Relevanz neuromuskuloskeletaler Nebenwirkungen zeigt sich allein schon am Beispiel der zur Cholesterinsenkung eingesetzten Statine ( $\bullet$ Tab. 1). Fast fünf Millionen Menschen werden in Deutschland damit behandelt. Fünf bis zehn Prozent dieser Patienten bekommen Muskelbeschwerden [12].

Ergibt sich aus der Vorgeschichte oder aus dem Behandlungsverlauf der Verdacht auf medikamentenbedingte Beschwerden des Bewegungsapparates, informiert der Therapeut idealerweise den Arzt, mit dem er entsprechend der Heilmittelrichtlinie eng zusammenarbeitet [8].

Drei Fallbeispiele zeigen die Komplexität der Nebenwirkungsproblematik neuromuskuloskeletaler Beschwerden, die erst über mehrere Änderungen der Arbeitshypothesen auf ein Medikament zurückgeführt werden konnten.

\section{Fall 1: Myalgien}

Die 80-jährige Frau K. klagt über Schwäche und Schmerzen in beiden Oberschenkeln. Sie bezeichnet die Beschwerden als Muskelkatergefühl oder auch Ermüdungsschmerz, der besonders beim Bergaufgehen oder Treppensteigen auftritt und ihre Gehstrecke einschränkt ( Abb. 1). In ihrer Wohnsiedlung gibt es nur noch ein Ladengeschäft, das sie selbstständig erreichen kann, eine kleine Metzgerei. Diese ist einhundert Meter von ihrem Wohnhaus entfernt. Wenn sie ihre Schmerzen hat, kann sie die teilweise ansteigende Strecke nicht

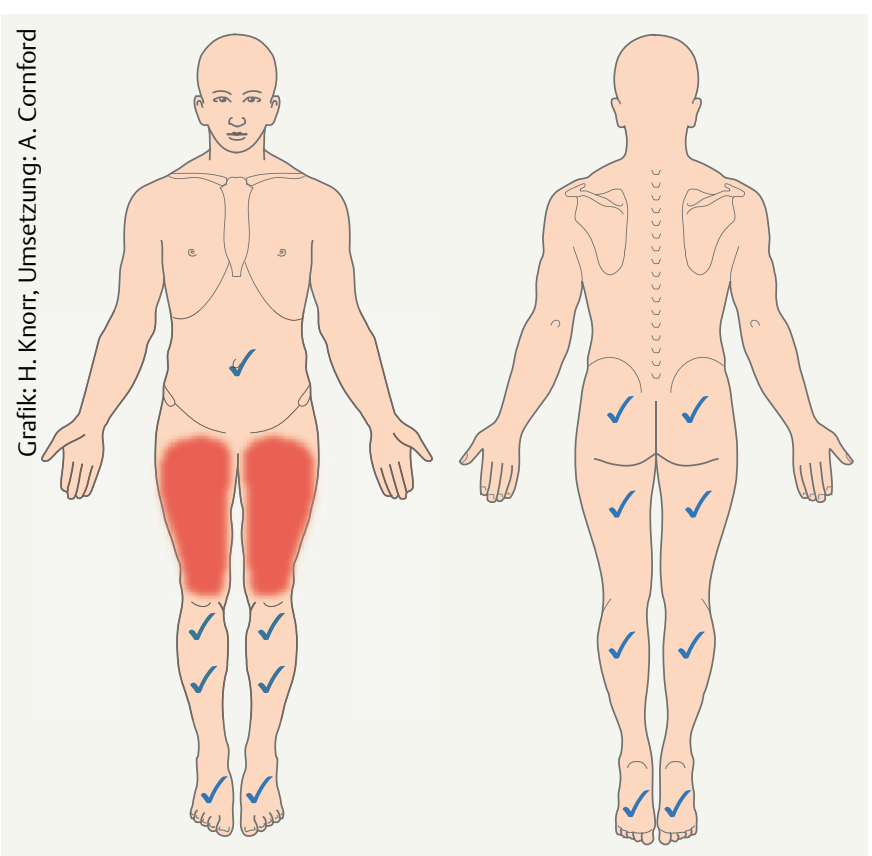

Abb. 1 Bodychart Frau K. 
mehr bewältigen. Das Problem wird noch dadurch verschärft, dass neben der Metzgerei die von ihr gern aufgesuchte Friseurin arbeitet.

\section{Erste Arbeitshypothese (des Hausarztes)}

Frau K. hat eine koronare Herzkrankheit. Vor sechs Jahren erhielt sie einen aortokoronaren Venenbypass und eine künstliche Aortenklappe aufgrund einer Aortenstenose. Ihre kardiovaskulären Risikofaktoren sind Hypercholesterinämie und arterieller Hypertonus. Wenn die Herzkranzgefäße arteriosklerotisch verengt sind, könnten auch die Becken-Bein-Arterien betroffen sein. Leidet Frau K. an einer peripheren arteriellen Verschlusskrankheit mit einer dafür typischen Claudicatio intermittens? Zwingt sie also ein Ischämieschmerz der Oberschenkelmuskulatur zum Anhalten? Diese Fragestellung wird von ihrem Kardiologen bearbeitet, den sie jährlich zur echokardiographischen Kontrolle ihres biologischen Aortenklappenersatzes kontaktiert. Im Belastungs-EKG erreicht sie maximal 50 Watt, der Abbruch erfolgt wegen Beinschmerzen. Da jedoch der Knöchel-Arm-Index, der Quotient aus dem systolischen Blutdruck von Unterschenkel und Oberarm, mit 1,1 in der Norm ist, liegt kein Hinweis für eine periphere arterielle Verschlusskrankheit als Ursache für die Oberschenkelbeschwerden vor.

\section{Zweite Arbeitshypothese (des Neurologen)}

Frau K. wird nun zum Neurologen überwiesen. Dessen klinischer Untersuchungsbefund ist unauffällig, insbesondere liegen keine Paresen vor. Das EMG zeigt keine myopathischen Potentiale, auch liegt keine für viele Muskelerkrankungen typische Kreatinkinase-Erhöhung vor. Aus früherer Bildgebung war bekannt, dass Frau K. degenerative Veränderungen der Wirbelsäule aufweist. So litt sie auch immer wieder unter Lumbalgien und zervikogenen Kopfschmerzen. Engen zum Beispiel spondylotische Randzacken oder spondylarthrotische Auftreibungen den lumbalen Spinalkanal oder die Intervertebralforamina ein? Hat Frau K. die für eine lumbale Spinalkanalstenose typische Claudicatio spinalis? Sind also radikulopathische Schmerzen der L3/L4-versorgten Oberschenkelregion für die Einschränkung ihrer Gehstrecke verantwortlich? Das daraufhin angefertigte MRT der LWS zeigt einen normal weiten Spinalkanal und nur diskrete Forameneinengungen ohne Bedrängung nervaler Strukturen. Eine Claudicatio spinalis ist damit unwahrscheinlich. Das klinische Bild mit Schmerzen beim Bergaufgehen spricht auch dagegen.

\section{Dritte Arbeitshypothese (des neuromuskulären Zentrums)}

Der Neurologe stellt Frau K. nun im neuromuskulären Zentrum einer neurologischen Klinik in der $25 \mathrm{~km}$ entfernten Großstadt vor. Klinische und elektromyographische Untersuchung ergeben auch hier keinen pathologischen Befund. Frau K. nimmt seit vier Jahren täglich $20 \mathrm{mg}$ Simvastatin zur Cholesterinsenkung ein. Diese Cholesterinsynthese-Enzymhemmer haben mit einer Häufigkeit von über zehn Prozent Nebenwirkungen auf die Muskulatur, die sich durch Schmerzen und Schwäche äußern [6]. Besonders häufig ist die Oberschenkelmuskulatur betroffen [12].

Hat Frau K. solche Statin-assoziierten Muskelsymptome (SAMS)? Das neuromuskuläre Zentrum empfiehlt einen Auslassversuch des Statins. Bevor der Hausarzt sich zu einem solch einfachen Vorge-

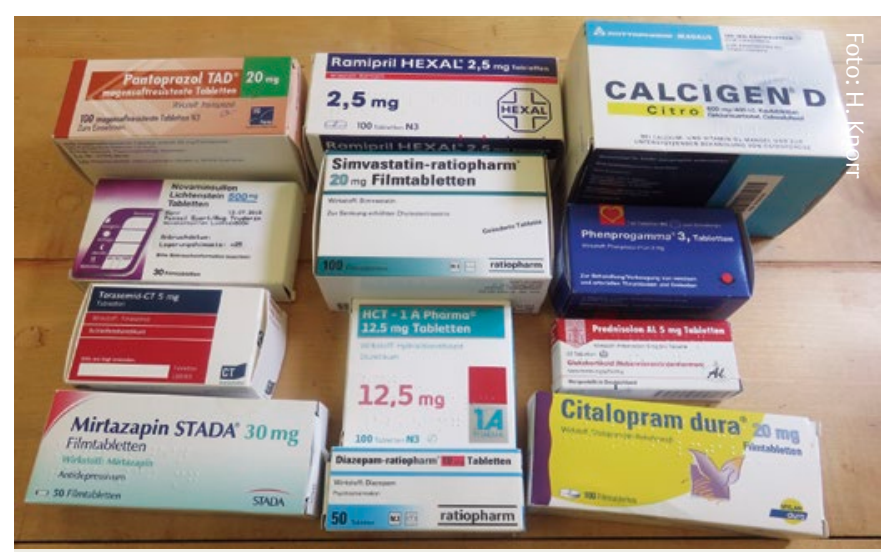

Abb. 2 Medikamente von Frau K., die sie täglich einnimmt. Außer Simvastatin können acht weitere Medikamente von Frau K. muskuläre Nebenwirkungen verursachen $(\downarrow$ Tab. 2 siehe Zusatzmaterial online).

hen entschließen kann, lässt er zuerst eine MR-Angiographie der Aorta abdominalis und Becken-Bein-Arterien anfertigen, die jedoch keinen Nachweis auf signifikante Gefäßstenosen zeigt. Eine Woche nach Absetzen des Simvastatins ist Frau K. beschwerdefrei, ohne Oberschenkelschmerzen, und wieder fähig, ihre alltagsrelevanten Gehstrecken zu bewältigen.

\section{Fazit von Fall 1 - Myalgien durch Cholesterinsenker Simvastatin}

Simvastatin ist das Medikament mit der höchsten Prävalenz von muskulären Nebenwirkungen nicht nur unter den Statinen, sondern unter allen gängigen Medikamenten. In der Nebenwirkungsdatenbank des BfArM werden Myalgie 511-mal, Myopathie/Myositis 125-mal, Rhabdomyolyse 330-mal und Muskelschwäche 79-mal, in der Nebenwirkungsdatenbank von sanego werden Muskelschmerzen 152-mal und Muskelschwäche 9-mal genannt. Diese Werte liegen zehn bis 50-mal höher als bei der ebenfalls als häufig eingestuften Kortison-Myopathie. Trotzdem wurde von ärztlicher Seite erst nach zwei Jahren ein Auslassversuch unternommen. Grund dafür könnte sein, dass das Markerenzym für Muskelerkrankungen, die Kreatinkinase (CK), in der Norm war.

Simvastatin kann Statin-assoziierte Muskelsymptome in drei Schweregraden verursachen [6]:

1. Myalgie: Schwäche und Schmerzen ohne CK-Erhöhung

2. Myopathie: Schwäche und Schmerzen mit CK-Erhöhung

3. Rhabdomyolyse: Schwäche und Schmerzen mit Muskelscha-

den und CK-Erhöhung über das Zehnfache der oberen Norm Frau K. hatte den niedrigsten Schweregrad. Nach vier Wochen wurde das mit weniger Nebenwirkungen behaftete Pravastatin in geringerer Dosis angesetzt. Muskelsymptome traten nicht wieder auf.

\section{Fall 2: Arthritis}

Der 60-jährige Herr K. wird von seiner Zahnärztin zum Kieferchirurgen überwiesen, der eine chirurgische Wurzelspitzenresektion am ersten oberen Backenzahn links durchführen soll. Der Wurzelkanal war schon vor sechs Jahren wegen rezidivierender 
Tab. 1 Häufigkeit von Statin-Verordnungen in Deutschland, Schwabe und Paffrath 2015 [14].

\begin{tabular}{|l|l|l|}
\hline Verordnungen von Statinen $\mathbf{2 0 1 4}$ & Verordnete Tagesdosen (DDD) in Mio. & Verordnungsanteil an Statinen \\
\hline Simvastatin & 1353 & $75 \%$ \\
\hline Atorvastatin & 324 & $18 \%$ \\
\hline Pravastatin & 76 & $4 \%$ \\
\hline Fluvastatin & 45 & $2,5 \%$ \\
\hline
\end{tabular}

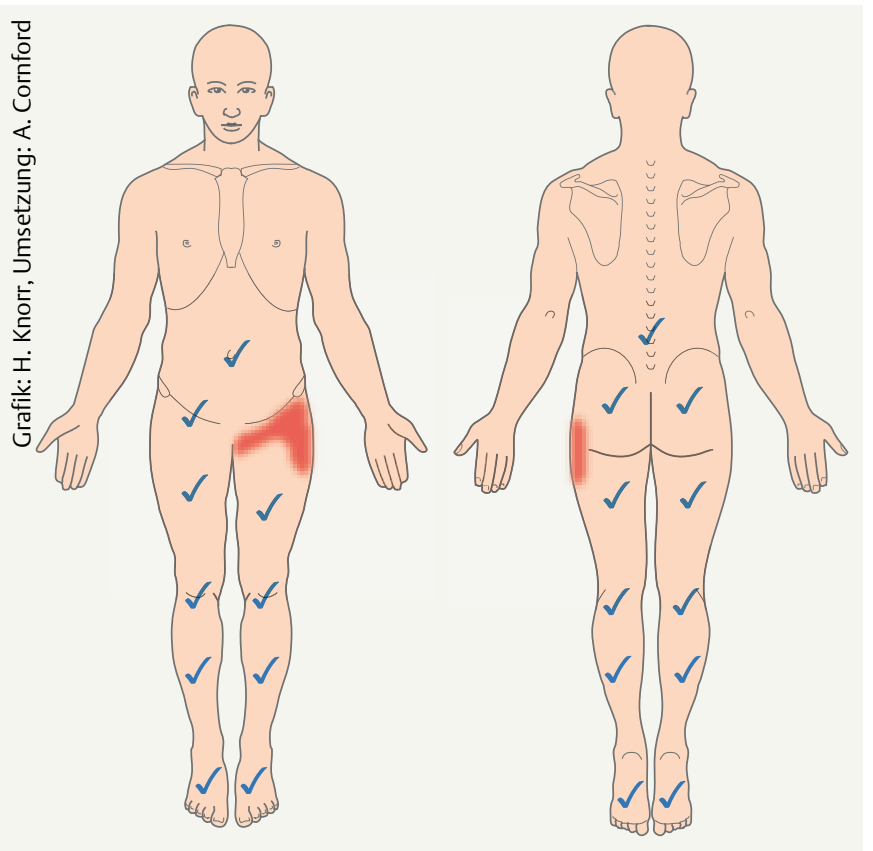

Abb. 3 Bodychart Herr K.

Zahnschmerzen bei Parodontose von der Zahnärztin nach mechanischer Zerstörung und Entfernung des Zahnmarks und damit des Zahnnervs mit einer Füllung langstreckig verschlossen worden. Nun hat sich oberhalb des Zahnes ein eitriger Herd um die Wurzelspitze gebildet. Zur Prophylaxe der intraoperativen Infektionsausdehnung und hämatogenen Streuung wird schon vor der Behandlung mit der Antibiose begonnen. Herr K. erhält Clindamycin, ein Lincosamid, 3-mal $600 \mathrm{mg}$ täglich für sieben Tage, erstmalig morgens vor dem Eingriff. Während der Operation stellt sich heraus, dass aufgrund der Ausdehnung des entzündlichen Areals der Zahn gezogen werden muss. Bei der Zahnextraktion entsteht ein kleines Loch zur benachbarten Kieferhöhle. Diese enthält polypenartige mukoide Vegetationen, die in der präoperativen Bildgebung deutlich sichtbar sind. Der Kieferchirurg entfernt dieses Gewächs durch die kleine Öffnung gleich mit. Schon zehn Jahre zuvor sind bei Herrn K. über einen transnasalen Zugang vom HNO-Arzt Polypen aus derselben Kieferhöhle extrahiert worden, die immer wieder Infekte der oberen Luftwege verursacht hatten.

Herr K. ist von dem unerwartet großen Eingriff, der unter lokaler Betäubung erfolgt, erschöpft. Am Wochenende, das auf den OP-Tag folgt, kann er sich ausruhen. Danach nimmt er seine Arbeit als praktischer Arzt wieder auf. In der Nacht des dritten postoperativen Tages wacht er um drei Uhr morgens mit dumpfen links- seitigen Hüftschmerzen (NRS 8/10) auf ( $\bullet$ Abb. 3). Nur mit einer Entlastungslagerung in leichter Hüftflexion kann er nach einer Stunde wieder einschlafen. Tagsüber ist der Schmerz zwar fast weg (NRS 2/10), Herr K. weist jedoch ein leicht hinkendes Gangbild auf. Eine Morgensteifigkeit besteht nicht.

\section{Erste Arbeitshypothese}

Da er ja am Wochenende ausgeruht hat, fällt Überlastung als mögliche Schmerzursache für ihn aus. Er hatte in letzter Zeit immer mal wieder Hüftschmerzen rechts (bei geringer Koxarthrose) oder Knieschmerzen links (bei ausgeprägter Gonarthrose) nach dem Joggen, das er zweimal wöchentlich ausübt. Eine aktivierte Koxarthrose links als Ursache seiner Beschwerden kommt daher für ihn nicht in Frage, da er noch nie in der linken Hüfte Beschwerden hatte. In der nächsten Nacht wacht er mit denselben Schmerzen, wieder um drei Uhr, auf.

\section{Zweite Arbeitshypothese}

Bewegungseinschränkung des linken Hüftgelenks (Innenrotation, Extension, Abduktion) und Leistendruckschmerz weisen auf eine Arthritis hin. Herr K. hat seit 35 Jahren eine schwach ausgeprägte Psoriasis. Nur einmal, vor drei Jahren, kam es zu einer Arthritis des distalen Interphalangealgelenkes des linken Mittelfingers. Diese deutete er damals als Psoriasis-Arthritis und verzichtete auf weitere Diagnostik. Unter 2-mal 50 mg Diclofenac war er damals nach drei Tagen wieder beschwerdefrei. Sollten seine jetzigen Koxalgien auch von der Psoriasis kommen? Er erkundigt sich im Internet und googelt noch in der Nacht. Seine Recherche ergibt: Koxarthritis wäre für Psoriasis eher ungewöhnlich [11].

\section{Dritte Arbeitshypothese}

Beim Grübeln über die Ursache seiner Beschwerden fällt sein Blick auf die Clindamycin-Tablettenschachtel, die auf dem Tisch liegt. Könnten seine Hüftschmerzen vom Antibiotikum verursacht sein? Im Beipackzettel steht als „sehr seltene“ (<1/10000) Nebenwirkung „Polyarthritis“. Andere Medikamente nimmt er nicht. Am nächsten Tag geht er zu einem befreundeten Orthopäden. Das Hüft-Ultraschallbild zeigt einen Erguss, die Entzündungswerte sind gering erhöht: C-reaktives Protein $8 \mathrm{mg} / \mathrm{l}$ (Norm $<5 \mathrm{mg} / \mathrm{l}$ ), BSG $27 \mathrm{~mm}$ (Norm < $20 \mathrm{~mm}$ ), Harnsäure normal und Rheumafaktor negativ.

Die Diagnose ist also eine Clindamycin-bedingte Koxarthritis links. Clindamycin wird abgesetzt, dafür eine Kombination aus dem $\beta$-Lactam-Antibiotikum Amoxicillin, einem Aminopenicillin, und Clavulansäure, einem $ß$-Lactamasehemmer, verordnet. Erneut wacht Herr K. nachts um drei Uhr wieder auf, dieses Mal hat er jedoch nur ein dumpfes Druckgefühl in der linken Hüfte 
(NRS 1/10) ohne Bewegungseinschränkung. Er schläft weiter und ist ohne weitere Maßnahmen in der Folgezeit beschwerdefrei.

\section{Fazit von Fall 2 - Arthritis unter zahnärztlicher Antibiose mit Clindamycin}

Eine Monarthritis ist eine sehr seltene Nebenwirkung von Clindamycin [1]. In der Nebenwirkungsdatenbank des BfArM werden Arthralgie 9-mal, Arthritis 11-mal (Monarthritis einmal) und Gelenkerguss/-schwellung 12-mal, in der Nebenwirkungsdatenbank von sanego werden Gelenkschmerzen 10-mal und Gelenkentzündung einmal genannt. Auch was selten ist, muss in Betracht gezogen werden. Zumal Clindamycin das von Zahnärzten in Deutschland mit Abstand am häufigsten verordnete Antibiotikum ist [10]. Dabei gilt es wegen seinem ungünstigen Nebenwirkungsprofil in allen Leitlinien hinter Amoxicillin nur als Reserveantibiotikum [2].

\section{Fall 3: Polyneuropathie}

Der 68-jährige Herr S. hat seit über zehn Jahren einen Tremor der Hände. Das Zittern ist teilweise so stark, dass er keinen handgeschriebenen Brief mehr verfassen kann. Die Tremorfrequenz beträgt circa $6 \mathrm{~Hz}$. Der Tremor tritt nur bei einhändigen Aktionen auf. Als passionierter Golfspieler ist er froh, dass sein beidhändig ausgeübter Golfsport damit nicht beeinträchtigt wird.

\section{Erste Arbeitshypothese}

Als Professor der Medizin war er viele Jahre in einem physiologischen Institut tätig. So kennt er sich gut mit dem sympathischen Nervensystem aus und weiß, dass manche Tremorarten gut durch $\beta$-Blocker beeinflussbar sind. Mit einem solchen Sympathikus-Antagonisten, mit Propranolol, konnte er seinen Tremor deutlich bessern. Da er bei sich keine Tremor verursachende neurologische Erkrankung erkennen konnte, ging er von einem essenziellen Tremor aus. Der essenzielle Tremor ist eine Form des Tremors, die ohne erkennbare neurologische Grunderkrankung auftritt. In letzter Zeit bemerkt Herr S. zusätzlich, dass er in der Straßenbahn während der Fahrt nicht mehr sicher gehen kann. Auch beim schnelleren Gehen fühlt er sich nicht mehr sicher und hat daher Mühe, die Straßenbahn an der Haltestelle rechtzeitig zu verlassen. Außerdem stolpert er häufiger. Bekannte sprechen ihn auf sein wackeliges Gangbild an.

\section{Zweite Arbeitshypothese}

Er sucht nun eine Neurologin auf. Aufgrund der koordinativen Gehstörung, des Tremors und des beim Gehen etwas nach vorn gebeugten Oberkörpers vermutet sie ein Parkinson-Syndrom. Sie veranlasst eine Single-Photon-Emissionscomputertomographie (SPECT) der Basalganglien. Eine pathologische Dopaminspeicherung bestätigt ihre Vermutung. Eineinhalb Jahre lang bekommt Herr S. dopaminhaltige Medikamente und verschiedene Dopaminagonisten. Seine Symptome bessern sich nicht. Im Gegenteil: Neue Symptome kommen hinzu. Herr S. bemerkt nun zusätzlich eine zunehmende Sensibilitätsstörung in beiden Fußsohlen, die schließlich strumpfförmig bis zu den Knien reicht ( Abb. 4). Er spürt Berührungen abgeschwächt, Kältereize sind schmerzhaft.

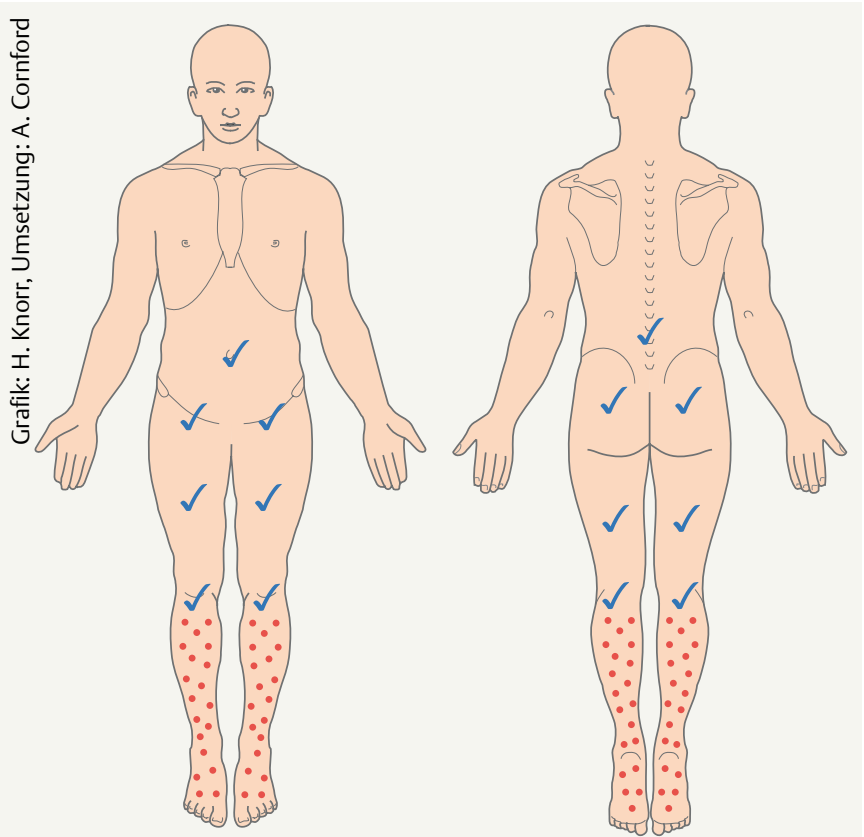

Abb. 4 Bodychart Herr S.

Als Universitätsprofessor war sein Spezialgebiet die Balneologie. Ein wichtiger Teil seines Lebens ist immer noch der Besuch von Kurorten mit Baden im Thermalwasser. Schon thermisch indifferentes Wasser von $34^{\circ} \mathrm{C}$ wird nun jedoch von ihm als schmerzhaft heiß empfunden. Äußerst schmerzhafte, blitzartig einschießende nächtliche Wadenkrämpfe bringen ihn um den Schlaf. Da er vier Mal in der Woche ins Fitnessstudio zum Ausdauer- und Krafttraining geht, wundert er sich außerdem über einen Muskelschwund an den Beinen.

\section{Dritte Arbeitshypothese}

Da kommt ihm der Zufall zu Hilfe. Schon seit seiner Assistenzarzt-Zeit litt er an Vorhofflimmern. Er hatte damals verschiedene Medikamente ausprobiert. Nur eines, das Antiarrhythmikum Flecainid, hat ihm geholfen. Hatte er Flecainid einmal probehalber weggelassen, hatte er prompt wieder Vorhofflimmern. Schon eine Reduktion seiner üblichen Tagesdosis von 3-mal $100 \mathrm{mg}$ auf 2-mal $100 \mathrm{mg}$ bewirkte Vorhofflimmern. Bei einer kardiologischen Routinekontrolle fällt nun im EKG ein bisher nicht bekannter kompletter Rechtsschenkelblock auf. Da der Kardiologe eine Herzerkrankung als Ursache der Reizleitungsstörung ausgeschlossen hat, gerät sein langjähriges Medikament Flecainid in Verdacht. Der Kardiologe rät zum Absetzen, zumal Flecainid schon seit über 20 Jahren wegen kardialer Todesfälle und schwerer Schädigungen anderer Organe nur noch als Reserve-Antiarrhythmikum gilt [3]. Auch weist er ihn auf die nervenschädigende Wirkung des Medikaments hin.

Hat Herr S. eine Polyneuropathie durch jahrzehntelange Einnahme von Flecainid in maximaler Tagesdosis entwickelt?

Herr S. lässt eine Katheterablation durchführen, bei der durch Hochfrequenzstrom Herzmuskelgewebe im linken Vorhof verödet wird, das für das Vorhofflimmern verantwortlich ist. Der Eingriff ist erfolgreich, Herr S. kann das Flecainid absetzen. Innerhalb eines Jahres bessern sich Tremor und die schmerzhaften Krämp- 
fe, nicht jedoch die Sensibilitätsstörungen. Der deshalb aufgesuchte Chefarzt einer neurologischen Klinik bestätigt die Diagnose Polyneuropathie klinisch (Fehlen des Achillessehnenreflexes, Hypästhesie in der Sensibilitätsprüfung, Minderung der Tiefensensibilität) und elektroneurographisch (Minderung der Nervenleitgeschwindigkeit). Über die weitere Prognose macht er keine Aussage.

\section{Fazit von Fall 3 - Polyneuropathie nach langjähriger medikamentöser Herzfrequenzregulierung mit Flecainid} Ein seit Jahrzehnten benutztes Medikament führte bei Herrn S. zur Schädigung peripherer Nerven. Die Neuropathie ist als Nebenwirkung bei Flecainid verzeichnet. In der Nebenwirkungsdatenbank des BfArM werden Neuropathie 3-mal, Hypo-/Parästhesie 8-mal und Dyskinesie/Ataxie 4-mal, in der von sanego Taubheitsgefühl und Kraftlosigkeit je einmal genannt. Trotzdem hat es seit Beginn der neurologischen Symptome wie Geh- und Sensibilitätsstörungen drei Jahre gedauert, bis das Medikament abgesetzt wurde. In den drei Jahren danach waren die Beschwerden nur teilweise reversibel.

Wenn nicht mal der sehr belesene, betroffene Medizinprofessor die Möglichkeit einer unerwünschten Arzneimittelwirkung als Auslöser seiner Symptomatik in Betracht zieht, wie sollen dann Physiotherapeuten, die er wegen seiner Beschwerden vielleicht kontaktiert, zu dieser Hypothese gelangen? Es wäre eigentlich ganz einfach: Medikamente erfragen (Flecainid war sein einziges Dauermedikament), den Beipackzettel des Medikaments Flecainid lesen, unter Nebenwirkungen die Symptome des Patienten finden: „Prickeln der Haut, Koordinationsprobleme, Schwierigkeiten bei Bewegungen, vermindertes Berührungsgefühl, Zittern, nervöse Störungen in Armen und Beinen, Krämpfe“ und den behandelten Arzt kontaktieren, um mit ihm Rücksprache zu halten. In der wissenschaftlichen Fachinformation von Flecainid finden sich im Nebenwirkungsteil die entsprechenden medizinischen Begriffe: „Erkrankungen des Nervensystems: Parästhesien, Ataxie, Hypästhesien, Tremor, periphere Neuropathie, Krämpfe, Dyskinesie“.

\section{Diskussion}

Nebenwirkungen sind definiert als „schädliche und unbeabsichtigte Reaktionen auf ein Arzneimittel“ [5]. Solche „unerwünschte Arzneimittelwirkungen (UAW)“ [4] treten in unterschiedlicher Häufigkeit auf.

Die Häufigkeit der unerwünschten Arzneimittelwirkungen ist unter anderem abhängig von:

- Pharmakodynamik und -kinetik,

- Dosierung,

- therapeutischer Breite (Dosisabstand zwischen Wirkung und Nebenwirkung),

- Arzneimittelinteraktionen des Medikaments und

- Patientenparametern wie:

- Compliance (Einnahme des Medikaments wie vorgeschrieben?),

- Funktion der Medikamenten-eliminierenden Organe (Leberinsuffizienz? Niereninsuffizienz?),
- genetischer Ausstattung (Aktivität abbauender Enzyme? Anzahl wirkungsauslösender Rezeptoren?

Aktivität ausscheidender Membrantransportsysteme?) oder

- Reaktion des Immunsystems (Allergie? Auslösung von Autoimmunreaktionen?).

:: Bei unklaren Symptomen des neuromuskulo-

skeletalen Systems sollte immer auch frühzeitig

an eine unerwünschte Arzneimittelwirkung gedacht

werden.

Das Thema Nebenwirkungen wird, wie in den Fällen dargestellt, noch komplexer dadurch, dass Medikamente häufig verabreicht werden, obwohl sie wegen Häufigkeit oder Schwere der Nebenwirkungen nur als Reservemittel vorgesehen sind. Die Nebenwirkungssymptome sind oft auch typische Zeichen von Krankheiten, die Therapeuten und Ärzte als klinische Muster abgespeichert haben. Es kann dadurch zu protrahierten Verläufen über mehrere Jahre kommen wie in Fall 1 und 3.

Tabelle 2 soll das Clinical Reasoning unterstützen (siehe $\mathrm{Zu}$ satzmaterial online). Sie enthält zum einen die am häufigsten verordneten und gekauften Medikamente, die neuromuskuloskeletale Nebenwirkungen verursachen. Zum anderen sind in der Tabelle Medikamente gelistet, die in der medizinischen Fachliteratur mit typischen neuromuskuloskeletalen Nebenwirkungen beschrieben sind. Es ist dabei zu beachten, dass in der medizinischen Fachliteratur oft eine deutlich höhere Häufigkeit der Nebenwirkungen angegeben wird als in den wissenschaftlichen Fachinformationen der Pharma-Industrie. Die angegebenen Häufigkeiten der Nebenwirkungen sind wie folgt definiert (siehe Zusatzmaterial online):

- sh $=$ sehr häufig $=\geq 1$ Behandelter von $10=\geq 10 \%$

- $\mathrm{h}$ = häufig = 1 bis 10 Behandelte von $100=\geq 1 \%$ bis $<10 \%$

- $\mathrm{g}=$ gelegentlich $=1$ bis 10 Behandelte von $1000=\geq 0,1 \%$ bis < $1 \%$

- $\mathrm{s}=$ selten $=1$ bis 10 Behandelte von $10000=\geq 0,01 \%$ bis $<0,1 \%$

- ss $=$ sehr selten = weniger als 1 Behandelter von $10000=<$ $0,01 \%$

- $\mathrm{nb}=$ nicht bekannt = Häufigkeit auf Grundlage der verfügbaren Daten nicht abschätzbar

FAZIT ○

Viele neuromuskuloskeletale Probleme können auch von einem Medikament verursacht werden. Physiotherapeuten sollten sich immer über die Medikation des Patienten informieren. Es ist sinnvoll, sich schon bei der Erhebung der Vorgeschichte die möglichen neuromuskuloskeletalen Nebenwirkungen der Medikamente des Patienten aus dem Beipackzettel zu notieren. Bei protrahiertem Therapieverlauf und bei Nicht-Übereinstimmung mit typischen problemrelevanten klinischen Mustern arbeiten Therapeuten mit dem zuständigen Arzt zusammen, indem sie ihn über den Therapieverlauf informieren 
und mit ihm kooperierend die Möglichkeit der Medikamentennebenwirkung als Problemursache überprüfen. Auslassversuche und Absetzung von Medikamenten sollen immer über den Arzt erfolgen. Nach Absetzen des Medikaments führt der Therapeut ein Re-Assessment durch, dessen Ergebnis zur Entscheidung der Fortsetzung oder Änderung der Medikation beiträgt. In allen Fragen der Medikation ist eine gute Zusammenarbeit mit dem Arzt wichtig.

\section{AUTOR}

Dr. med. Hansjörg Knorr ist Facharzt für Physikalische und Rehabilitative Medizin und ärztlicher Leiter der DVMT-e.V.Weiterbildung (Zertifikatsmodule). Er arbeitet zudem als Dozent im OMT-DVMT® Weiterbildungsprogramm. Dr. Knorr

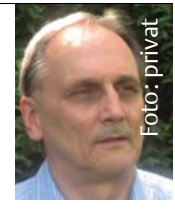
blickt auf eine langjährige rehabilitations- und allgemeinmedizinische Tätigkeit im Rehabilitationszentrum Pfennigparade München und notfallmedizinische Tätigkeit im kassenärztlichen Bereitschaftsdienst zurück.

Korrespondenzadresse:

Geroldseckstraße 4

81375 München

hansjoerg.knorr@t-online.de

\section{ZUSATZMATERIAL}

- Tabelle 2: Nebenwirkungen von Medikamenten auf den Bewegungsapparat, entnommen den wissenschaftlichen Fachinformationen des Fachinfo-Service ${ }^{\circledR}$ der Roten Liste ${ }^{\circledR}$ Service GmbH [7].

- Literaturverzeichnis

Zusatzmaterial am Ende der HTML-Version unter: www.thieme-connect.de/products/manuelletherapie
DOI 10.1055/s-0042-112278

manuelletherapie 2016; 20: 172-177

(c) Georg Thieme Verlag KG

Stuttgart · New York · ISSN 1433-2671

\section{BIBLIOGRAFIE}

